Article

\title{
Numerical Analysis, Circuit Simulation, and Control Synchronization of Fractional-Order Unified Chaotic System
}

\author{
Guohui Li *(D), Xiangyu Zhang and Hong Yang *(i) \\ School of Electronic Engineering, Xi'an University of Posts and Telecommunications, Xi'an, Shaanxi 710121, \\ China; zxy960819@stu.xupt.edu.cn \\ * Correspondence: liguohui@xupt.edu.cn (G.L.); yanghong@xupt.edu.cn (H.Y.); \\ Tel.: +86-29-8816-6273 (G.L. \& H.Y.)
}

Received: 20 October 2019; Accepted: 5 November 2019; Published: 8 November 2019

\begin{abstract}
The traditional method of solving fractional chaotic system has the problem of low precision and is computationally cumbersome. In this paper, different fractional-order calculus solutions, the Adams prediction-correction method, the Adomian decomposition method and the improved Adomian decomposition method, are applied to the numerical analysis of the fractional-order unified chaotic system. The result shows that different methods have higher precision, smaller computational complexity, and shorter running time, in which the improved Adomian decomposition method works best. Then, based on the fractional-order chaotic circuit design theory, the circuit diagram of fractional-order unified chaotic system is designed. The result shows that the circuit simulation diagram of fractional-order unified chaotic system is basically consistent with the phase space diagram obtained from the numerical solution of the system, which verifies the existence of the fractional-order unified chaotic system of 0.9-order. Finally, the active control method is used to control and synchronize in the fractional-order unified chaotic system, and the experiment result shows that the method can achieve synchronization in a shorter time and has a better control performance.
\end{abstract}

Keywords: fractional-order chaotic system; Adams prediction-correction method; Adomian decomposition method; fractional-order chaotic circuit; active control method

\section{Introduction}

Since the definition of the Lorenz system by Lorenz in 1963, the study of modern chaos theory has made rapid progress. The typical ones are Chen system [1], Lü system [2], Rössler system [3], generalized Lü system [4], etc. In 2003, Liu et al. [5] discovered a chaos system that can produce four helical chaotic attractors in a three-dimensional chaos system. In 2004, Liu et al. [6] discovered a class of three-dimensional chaotic system with square terms. In 2005, Qi et al. [7] added a nonlinear term on the basis of Lorenz system and obtained a new chaotic system. In 2009, Bao et al. [8] proposed a three-dimensional continuous autonomous chaotic system with simple equations and complex attractor topologies.

The dynamic characteristics of fractional-order chaotic system is related to the corresponding order. In the same chaotic system, when the selection of order is different, the corresponding chaotic characteristics, such as bifurcation graph and Lyapunov exponent graph, are also different, so they have more abundant chaotic characteristics. Scholars at home and abroad have carried out this research. Chen et al. [9], Wang et al. [10] studied the chaotic circuit of fractional-order Liu system and other fractional-order chaotic system derived from fractional-order Liu system. Mao et al. [11], He et al. [12] studied fractional-order Lorenz system and circuit simulation. At the same time, the control and 
synchronization of fractional-order chaotic system also has made progress. $\mathrm{Wu}$ [13] proposed a new control method based on linear matrix inequality to solve the synchronization of two fractional-order chaotic system. Yan et al. [14] proposed a new fractional-order sliding mode controller to solve the synchronization problem of uncertain fractional-order chaotic system with modeling errors and external disturbances. Rabah et al. [15] designed a new robust fractional-order proportional integral-differential (FOPID) controller. Rajagopal et al. [16] derived and obtained a four-dimensional fractional-order discrete power model. Although some achievements have been made in these studies, the traditional fractional-order chaotic system solution methods for example calling function method have some problems, such as low accuracy and computationally cumbersome.

Based on these problems, in this paper, different methods are used to solve the numerical solution of the fractional-order unified chaotic system, and the phase space diagram of the corresponding order is obtained. Then, the circuit simulation diagram of the chaotic system is designed. Finally, three control laws are obtained by the active control method, and the control and synchronization of the fractional-order unified chaotic system are completed.

\section{Intege-Order Unified Chaotic System}

The mathematical model of the three-dimensional continuous chaotic system [17] is as follows:

$$
\left\{\begin{array}{l}
\frac{d x}{d t}=(25 \alpha+10)(y-x) \\
\frac{d y}{d t}=(28-35 \alpha) x-x z+(29 \alpha-1) y \\
\frac{d z}{d t}=x y-\frac{(8+\alpha) z}{3}
\end{array}\right.
$$

Since the chaotic system can be transformed among Lorenz system, Chen system, and Lü system when $\alpha$ takes different value, the system is called unified chaotic system. When $\alpha \in[0,1]$, the system has a global chaotic characteristic. The attractor diagram for $\alpha$ with different values is shown in Figure 1 . Time domain waveform is shown in Figure 2. Since the parameter $\alpha$ takes different value and the system corresponds to a different three-dimensional continuous chaotic system. When $\alpha \in[0.8,1]$, the Lyapunov exponent diagram is listed, as shown in Figure 3. The maximum Lyapunov exponent is greater than zero. It indicates that the system is in a chaotic state.

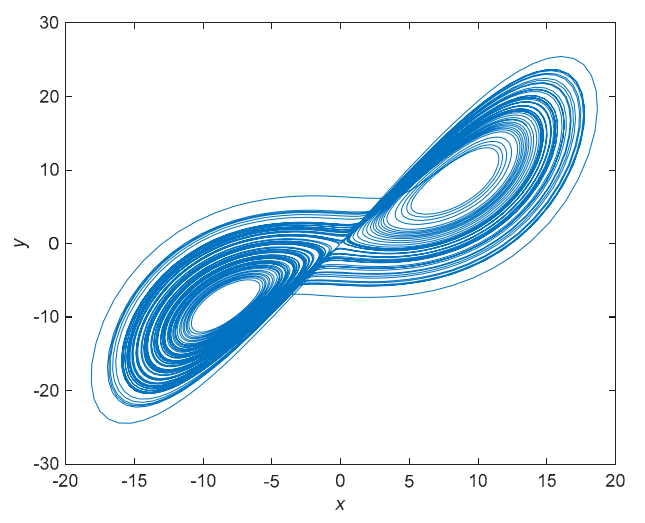

(a)

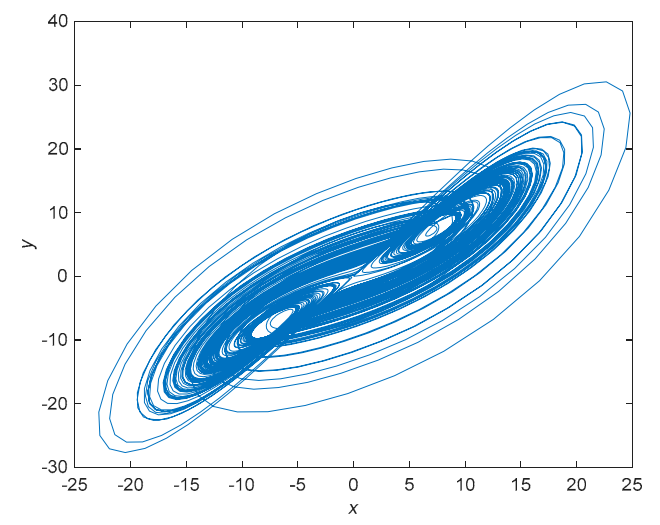

(b)

Figure 1. Cont. 


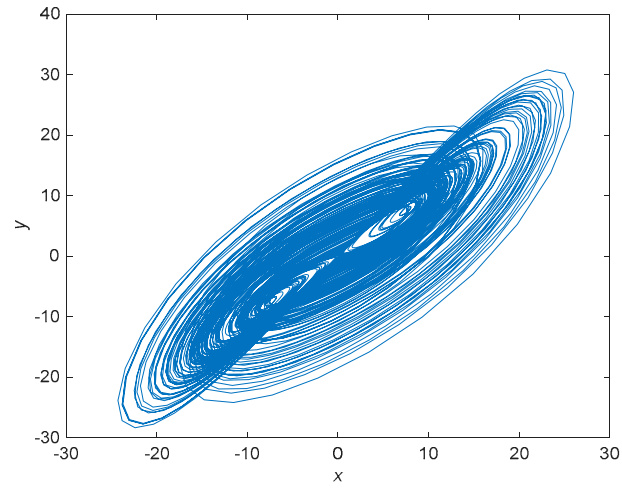

(c)



(d)

Figure 1. Phase space diagram of $x$-y plane. (a) $\alpha=0 ;(\mathbf{b}) \alpha=0.5 ;$ (c) $\alpha=0.8 ;(\mathbf{d}) \alpha=1$.

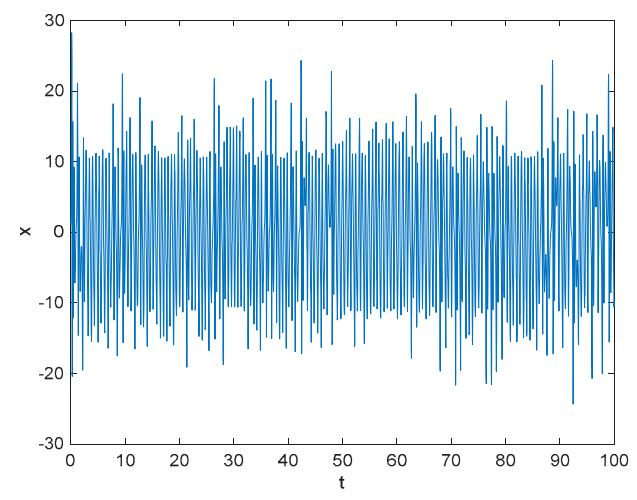

(a)

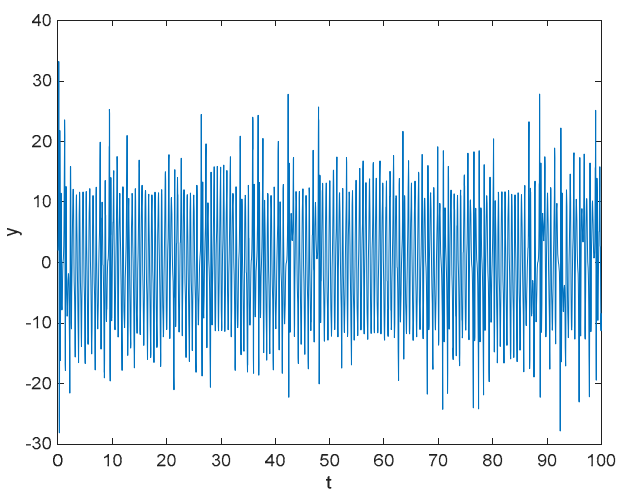

(b)

Figure 2. Time domain waveform of integer-order unified chaotic system with $\alpha=1$. (a) $x(t)$; (b) $y(t)$.

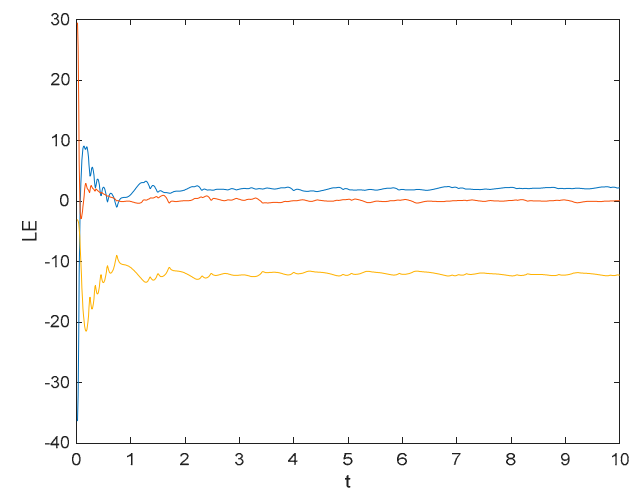

Figure 3. Lyapunov exponent of integral-order unified chaotic system with $\alpha \in[0.8,1]$.

\section{Fractional-Order Unified Chaotic System}

\subsection{Common Definitions of Fractional Calculus}

(1) The Grünwald-Letnikov fractional differential is defined as [18]:

$$
{ }_{a} D_{t}^{q} f(t)=\frac{d^{q} f(t)}{d(t-a)^{q}}=\lim _{N \rightarrow \infty}\left[\frac{t-a}{N}\right]^{-q} \sum_{j=0}^{N-1}(-1)^{j}\left(\begin{array}{c}
q \\
j
\end{array}\right) f\left(t-j\left[\frac{t-a}{N}\right]\right)
$$


(2) The Riemann-Liouville fractional differential is defined as [18]:

$$
{ }_{a} D_{t}^{q} f(t)= \begin{cases}\frac{1}{\Gamma(-q)} \int_{a}^{t}(t-\tau)^{-q-1} f(\tau) d(\tau), & q<0 \\ (t), & q=0 \\ \left.D^{n}{ }_{{ }_{a}} D^{q-n} f(t)\right], & q>0\end{cases}
$$

where $\Gamma($.$) is a Gamma function, which is defined as:$

$$
\Gamma(x)=\int_{0}^{\infty} t^{x-1} e^{-t} d t
$$

(3) The Caputo fractional differential is defined as [18]:

$$
{ }_{a} D_{t}^{q} f(t)=\left\{\begin{array}{lr}
\frac{1}{\Gamma(m-q)} \int_{0}^{t} \frac{f(m)(\tau) d \tau}{(t-\tau)^{q+1-m}}, & m-1<q<m \\
\frac{d^{m}}{d t^{m}} f(t), & q=m
\end{array}\right.
$$

where the $q$-order differential and the power function respectively are:

$$
\begin{gathered}
D_{t_{0}}^{q} t^{r}=\frac{\Gamma(r+1)}{\Gamma(r+1-q)}\left(t-t_{0}\right)^{r-q} \\
D_{t_{0}}^{q} C=0
\end{gathered}
$$

\subsection{The Calling Function Method}

Redefine the mathematical model of the fractional-order unified chaotic system:

$$
\left\{\begin{array}{l}
D^{q 1} x_{1}=(25 \alpha+10)\left(x_{2}-x_{1}\right) \\
D^{q 2} x_{2}=(28-35 \alpha) x_{1}-x_{1} x_{3}+(29 \alpha-1) x_{2} \\
D^{q^{3}} x_{3}=x_{1} x_{2}-(8+\alpha) x_{3} / 3
\end{array}\right.
$$

When $\alpha=0$, construct the MATLAB function for the fractional-order unified chaotic system: Function $[\mathrm{T} \mathrm{Y}]=$ FOLorenz (parameters, orders, TSim, Y0), where parameters are system parameters, orders are derivative order, TSim is simulation time, and $Y 0$ is initial condition.

When $q=0.9, \mathrm{TSim}=100, \mathrm{Y} 0=[0.10 .10 .1]$, the phase space diagram is shown in Figure 4.

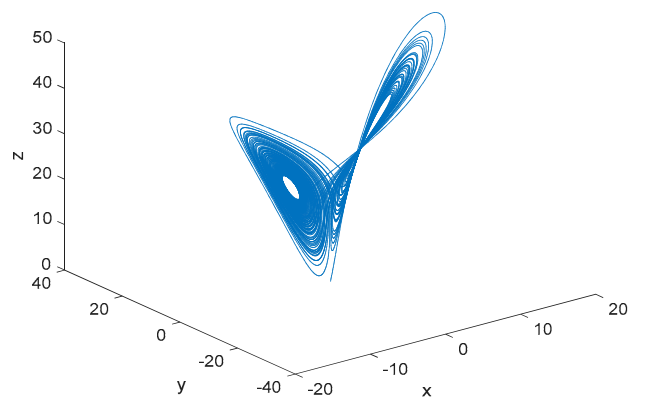

(a)

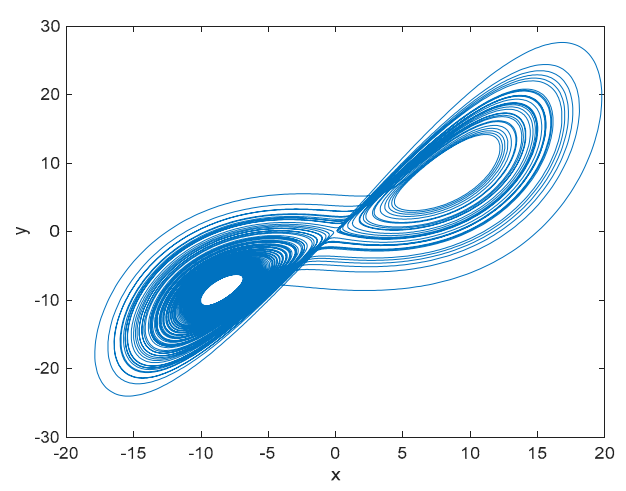

(b)

Figure 4. Cont. 


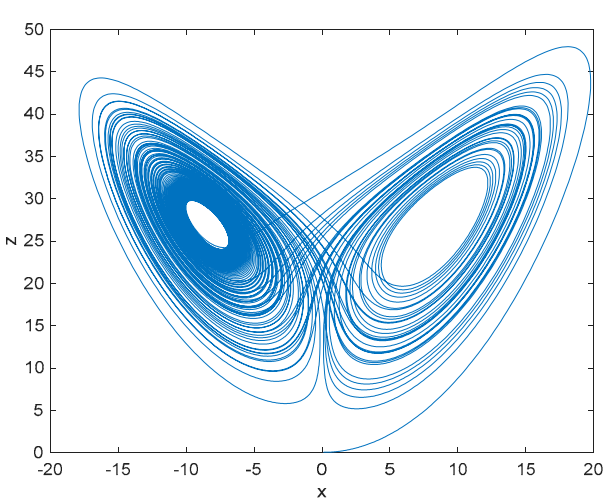

(c)

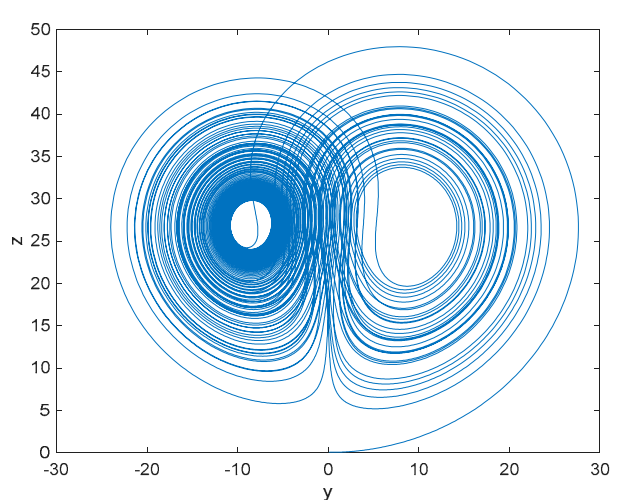

(d)

Figure 4. Phase space diagram of each plane. (a) $x-y-z$ plane; (b) $x-y$ plane; (c) $x-z$ plane; (d) $y-z$ plane.

3.3. The Adams-Bashforth-Moulton Prediction-Correction Method [19]

For the following differential equations:

$$
\left\{\begin{array}{lr}
\frac{d^{q} x}{d t^{q}}=f(t, x), & 0 \leq t \leq T \\
x^{k}(0)=x_{0}^{(k)}, & k=0,1,2,3, \ldots,\lceil q\rceil-1
\end{array}\right.
$$

Equivalent to the Volterra integral equation:

$$
x(t)=\sum_{k=0}^{n-1} x_{0}^{(k)} \frac{t^{k}}{k !}+\frac{1}{\Gamma(q)} \int_{0}^{t}(t-\tau)^{q-1} f(\tau, x(t)) d \tau
$$

The correction formula is:

$$
x_{h}\left(t_{n+1}\right)=\sum_{k=0}^{\lceil q\rceil-1} x_{0}^{(k)} \frac{t_{n+1}^{k}}{k !}+\frac{h^{q}}{\Gamma(q+2)} f\left(t_{n+1}, x_{h}^{p} t(n+1)\right)+\frac{h^{q}}{\Gamma(q+2)} \sum_{j=0}^{n} a_{j, n+1} f\left(t_{j}, x_{h}(t, j)\right)
$$

where,

$$
a_{j, n+1}= \begin{cases}n^{q+1}-(n-q)(n+1)^{q}, & j=0 \\ (n-j-2)^{q+1}+(n-j)^{q+1}-2(n-j+1)^{q+1}, & 1 \leq j \leq n\end{cases}
$$

The prediction formula is:

$$
x_{h}^{p}\left(t_{n+1}\right)=\sum_{k=0}^{n-1} x_{0}^{(k)} \frac{t_{n+1}^{k}}{k !}+\frac{1}{\Gamma(q)} \sum_{j=0}^{n} b_{j, n+1} f\left(t_{j}, x_{h}\left(t_{j}\right)\right)
$$

where,

$$
b_{j, n+1}=\frac{h^{q}}{q}\left((n-j+1)^{q}-(n-j)^{q}\right), 0 \leq j \leq n
$$

The basic algorithm of Adams-Bashforth-Moulton prediction-correction method is shown in Equations (11) and (13), where the coefficients are shown in Equations (12) and (14). Although the calculation of the fractional time domain analysis method is more complicated than the frequency domain method, the order of the calculus operator can calculate the step change smaller, which is more conducive to analyze the dynamics of the fractional system.

Now redefine the mathematical model of the fractional-order unified chaotic system:

$$
\left\{\begin{array}{l}
D^{q 1} x_{1}=(25 \alpha+10)\left(x_{2}-x_{1}\right) \\
D^{q 2} x_{2}=(28-35 \alpha) x_{1}-x_{1} x_{3}+(29 \alpha-1) x_{2} \\
D^{q 3} x_{3}=x_{1} x_{2}-(8+\alpha) x_{3} / 3
\end{array}\right.
$$


The discretization of fractional-order unified chaotic system can be obtained:

$$
\left\{\begin{aligned}
x_{n+1}= & x_{0}+\frac{(25 \alpha+10) h^{q}}{\Gamma(q+2)}\left(y_{n+1}^{*}-x_{n+1}^{*}+\sum_{j=0}^{n} a_{j, n+1} *\left(y_{j}-x_{j}\right)\right) \\
y_{n+1}= & y_{0}+\frac{h^{q}}{\Gamma(q+2)}\left(\left((28-35 \alpha) x_{n+1}^{*}-x_{n+1}^{*} z_{n+1}^{*}+(29 \alpha-1) y_{n+1}^{*}\right)+\sum_{j=0}^{n} a_{j, n+1}\right. \\
& \left.*\left((28-35 \alpha) x_{j}-x_{j} z_{j}+(29 \alpha-1) y_{j}\right)\right) \\
z_{n+1}= & z_{0}+\frac{h^{q}}{\Gamma(q+2)}\left(x_{n+1}^{*} y_{n+1}^{*}-(8+\alpha) z_{n+1}^{*} / 3+\sum_{j=0}^{n} a_{j, n+1} *\left(x_{j} y_{j}-(8+\alpha) z_{j} / 3\right)\right)
\end{aligned}\right.
$$

The steps to solve fractional differential equations by the Adams prediction-correction method are as follows:

Step 1: Determine the step size and the interval of solution. Taking the fractional-order unified chaotic system as an example, the step size $h=0.01$ is selected. The number of points is selected as $N=10,000$, and the upper limit of integration is $T=100(h=T / N)$.

Step 2: Calculate the predictive factor.

Step 3: Calculate the predictive value.

Step 4: Calculate the correction factor.

Step 5: Calculate the correction value.

Step 6: Determine if the end condition is meet.

Step 7: End the calculation if the condition is meet, or return to step 3.

The flow of numerical calculation of the Adams prediction-correction method is shown in Figure 5.

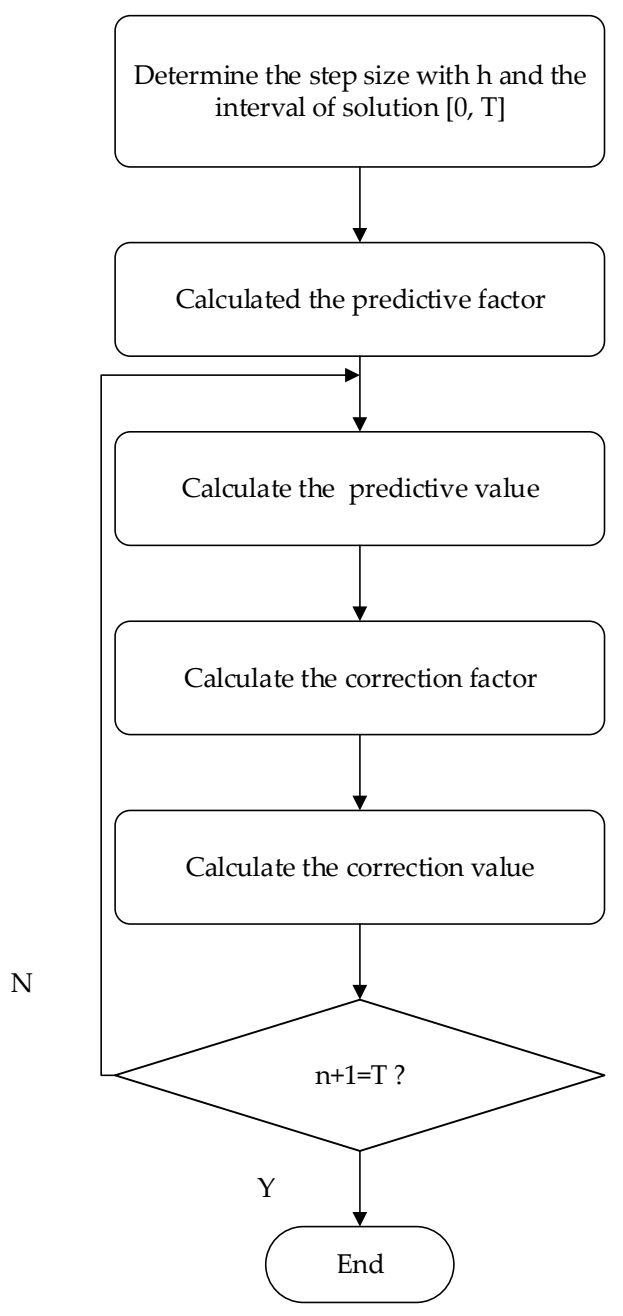

Figure 5. The flow of numerical calculation of the Adams prediction-correction method. 
When $q 1=q 2=q 3=0.9$, step length $h=0.001$, and $\alpha=0.8$, the phase space diagram of each plane is shown in Figure 6. When $q 1=q 2=q 3=0.9$, step length $h=0.001$, and $\alpha=1$, the phase space diagram of each plane is shown in Figure 7.

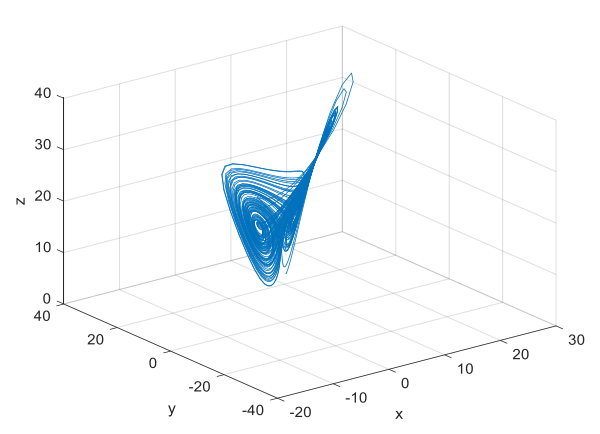

(a)

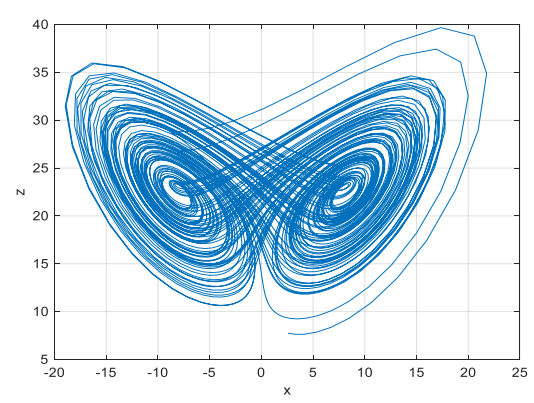

(c)

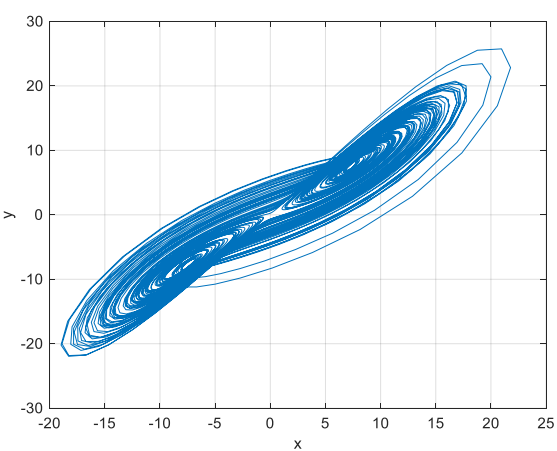

(b)

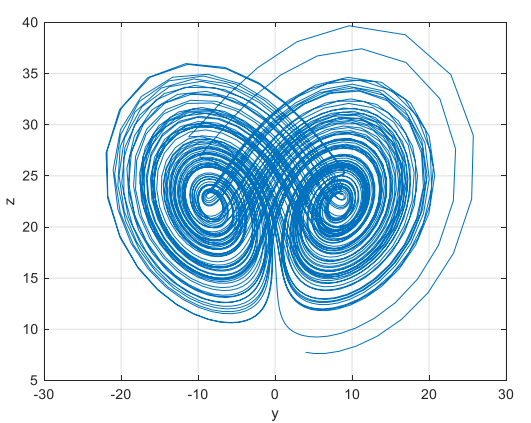

(d)

Figure 6. Phase space diagram of each plane when $\alpha=0.8$. (a) $x-y-z$ plane; (b) $x-y$ plane; (c) $x-z$ plane; (d) $y$-z plane.

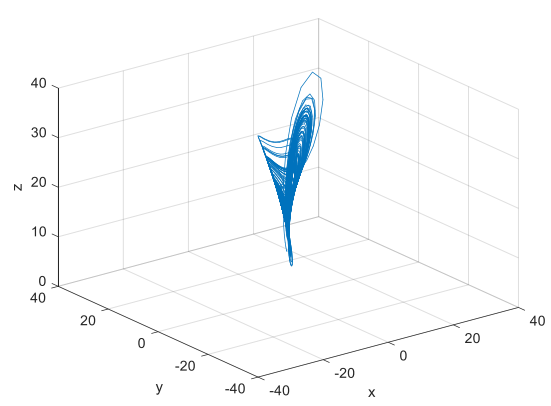

(a)

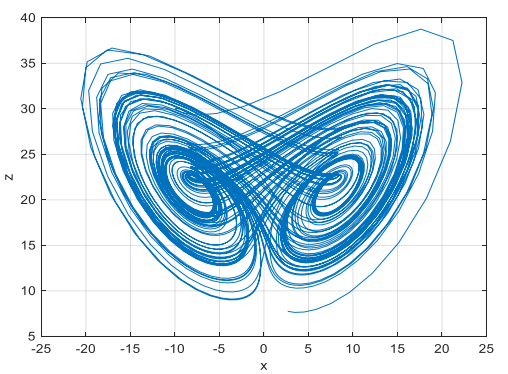

(c)

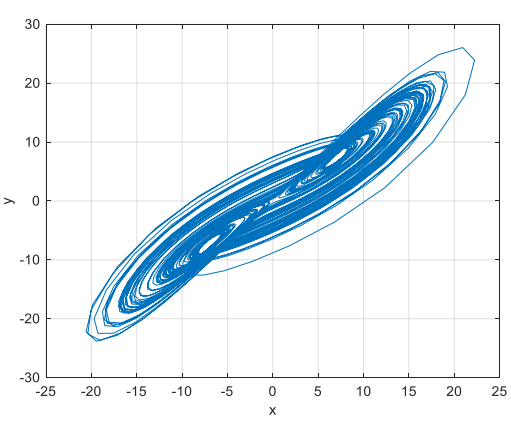

(b)

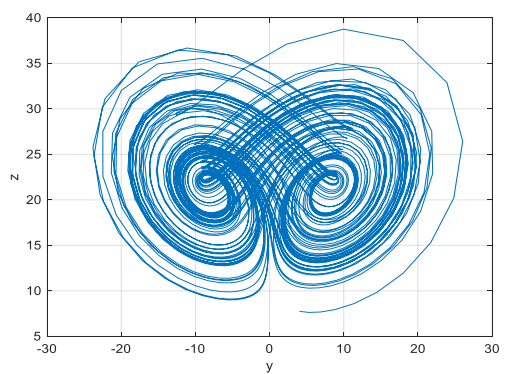

(d)

Figure 7. Phase space diagram of each plane when $\alpha=1$. (a) $x-y-z$ plane; (b) $x-y$ plane; (c) $x-z$ plane, (d) $y$-z plane. 
In addition, according to the Adams prediction-correction method, the phase space of the fractional-order Duffing oscillator when $q 1=q 2=q 3=0.98$ is solved, as shown in Figure 8. As a result, the Adams prediction-correction method is applicable to not only fractional-order unified chaotic system, but also other fractional-order chaotic system, such as fractional-order Duffing system.

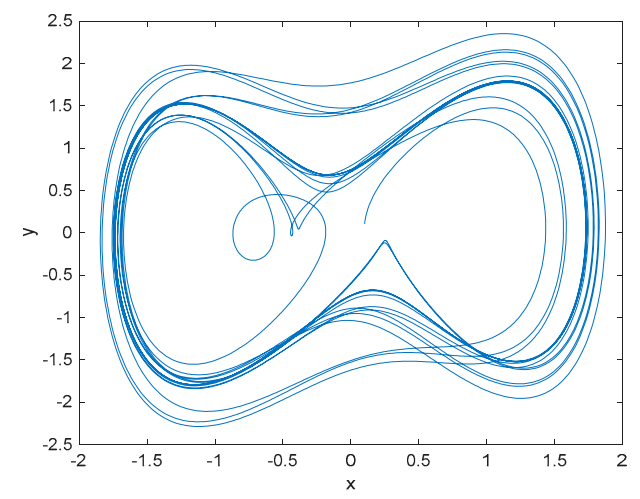

Figure 8. Duffing oscillator phase space diagram when $q 1=q 2=q 3=0.98$.

\subsection{The Adomian Decomposition Method}

The Adomian decomposition method [20] is a time-domain approximation algorithm that is suitable for the solution of fractional-order system. It is a time-domain solution algorithm that can deal with linear and non-linear problems. It is not necessary to carry out the discretization and a large amount of computer memory, and provide a high-precision approximate numerical solution.

The mathematical model of the fractional-order unified chaotic system is:

$$
\left\{\begin{array}{l}
D^{q 1} x_{1}=(25 \alpha+10)\left(x_{2}-x_{1}\right) \\
D^{q 2} x_{2}=(28-35 \alpha) x_{1}-x_{1} x_{3}+(29 \alpha-1) x_{2} \\
D^{q 3} x_{3}=x_{1} x_{2}-(8+\alpha) x_{3} / 3
\end{array}\right.
$$

By decomposing the linear and nonlinear terms in the system, we can obtain:

$$
\begin{aligned}
& {\left[\begin{array}{l}
L_{x 1} \\
L_{x 2} \\
L_{x 3}
\end{array}\right]=\left[\begin{array}{c}
(25 \alpha+10)\left(x_{2}-x_{1}\right) \\
(28-35 \alpha) x_{1}+(29 \alpha-1) x_{2} \\
-\frac{(8+\alpha)}{3} x_{3}
\end{array}\right] } \\
& {\left[\begin{array}{c}
N_{x 1} \\
N_{x 2} \\
N_{x 3}
\end{array}\right]=\left[\begin{array}{c}
0 \\
-x_{1} x_{3} \\
x_{1} x_{2}
\end{array}\right] }
\end{aligned}
$$

The nonlinear terms are decomposed and the previous seven items are selected. The following equation can be obtained:

$$
\left\{\begin{array}{l}
A_{2}^{0}=-x_{1}^{0} x_{3}^{0} \\
A_{2}^{1}=-x_{1}^{1} x_{3}^{0}-x_{1}^{0} x_{3}^{1} \\
A_{2}^{2}=-x_{1}^{2} x_{3}^{0}-x_{1}^{1} x_{3}^{1}-x_{1}^{0} x_{3}^{2} \\
A_{2}^{3}=-x_{1}^{3} x_{3}^{0}-x_{1}^{2} x_{3}^{1}-x_{1}^{1} x_{3}^{2}-x_{1}^{0} x_{3}^{3} \\
A_{2}^{4}=-x_{1}^{4} x_{3}^{0}-x_{1}^{3} x_{3}^{1}-x_{1}^{2} x_{3}^{2}-x_{1}^{1} x_{3}^{3}-x_{1}^{0} x_{3}^{4} \\
A_{2}^{5}=-x_{1}^{5} x_{3}^{0}-x_{1}^{4} x_{3}^{1}-x_{1}^{3} x_{3}^{2}-x_{1}^{2} x_{3}^{3}-x_{1}^{1} x_{3}^{4}-x_{1}^{0} x_{3}^{5}
\end{array}\right.
$$




$$
\left\{\begin{array}{l}
A_{3}^{0}=x_{1}^{0} x_{2}^{0} \\
A_{3}^{1}=x_{1}^{1} x_{2}^{0}+x_{1}^{0} x_{2}^{1} \\
A_{3}^{2}=x_{1}^{2} x_{2}^{0}+x_{1}^{1} x_{2}^{1}+x_{1}^{0} x_{2}^{2} \\
A_{3}^{3}=x_{1}^{3} x_{2}^{0}+x_{1}^{2} x_{2}^{1}+x_{1}^{1} x_{2}^{2}+x_{1}^{0} x_{2}^{3} \\
A_{3}^{4}=x_{1}^{4} x_{2}^{0}+x_{1}^{3} x_{2}^{1}+x_{1}^{2} x_{2}^{2}+x_{1}^{1} x_{2}^{3}+x_{1}^{0} x_{2}^{4} \\
A_{3}^{5}=x_{1}^{5} x_{2}^{0}+x_{1}^{4} x_{2}^{1}+x_{1}^{3} x_{2}^{2}+x_{1}^{2} x_{2}^{3}+x_{1}^{1} x_{2}^{4}+x_{1}^{0} x_{2}^{5}
\end{array}\right.
$$

According to the given conditions, the following equation can be obtained:

$$
\left\{\begin{array}{l}
x_{1}^{0}=x_{1}\left(t_{0}\right) \\
x_{2}^{0}=x_{2}\left(t_{0}\right) \\
x_{3}^{0}=x_{3}\left(t_{0}\right)
\end{array}\right.
$$

Make $c_{1}^{0}=x_{1}^{0}, c_{2}^{0}=x_{2}^{0}, c_{3}^{0}=x_{3}^{0}$, the following equation can be obtained:

$$
\left\{\begin{aligned}
x_{1}^{1} & =\left[(25 \alpha+10)\left(c_{2}^{0}-c_{1}^{0}\right)\right] \frac{\left(t-t_{0}\right)^{q}}{\Gamma(q+1)} \\
x_{2}^{1} & =\left[(28-35 \alpha) c_{1}^{0}-c_{1}^{0} c_{3}^{0}+(29 \alpha-1) c_{2}^{0}\right] \frac{\left(t-t_{0}\right)^{q}}{\Gamma(q+1)} \\
x_{3}^{1} & =\left[c_{1}^{0} c_{2}^{0}-(8+\alpha) c_{3}^{0} / 3\right] \frac{\left(t-t_{0}\right)^{q}}{\Gamma(q+1)}
\end{aligned}\right.
$$

From the iteration relation, the other five coefficients of $x$ can be deduced as follows:

$$
\begin{gathered}
\left\{\begin{array}{l}
c_{1}^{2}=(25 \alpha+10)\left(c_{2}^{1}-c_{1}^{1}\right) \\
c_{2}^{2}=(28-35 \alpha) c_{1}^{1}+(29 \alpha-1) c_{2}^{1}-c_{1}^{1} c_{3}^{0}-c_{1}^{0} c_{3}^{1} \\
c_{3}^{2}=\left[-\frac{8+\alpha}{3}\right] c_{3}^{1}+c_{1}^{1} c_{2}^{0}+c_{1}^{0} c_{2}^{1}
\end{array}\right. \\
\left\{\begin{array}{l}
c_{1}^{3}=(25 \alpha+10)\left(c_{2}^{2}-c_{1}^{2}\right) \\
c_{2}^{3}=(28-35 \alpha) c_{1}^{2}+(29 \alpha-1) c_{2}^{2}-c_{1}^{2} c_{3}^{0}-c_{1}^{1} c_{3}^{1} \frac{\Gamma(2 q+1)}{\Gamma^{2}(q+1)}-c_{1}^{0} c_{3}^{2} \\
c_{3}^{3}=\left[-\frac{8+\alpha}{3}\right] c_{3}^{2}+c_{1}^{2} c_{2}^{0}+c_{1}^{1} c_{2} \frac{\Gamma(2 q+1)}{\Gamma^{2}(q+1)}+c_{1}^{0} c_{2}^{2}
\end{array}\right. \\
\left\{\begin{aligned}
c_{1}^{4}= & (25 \alpha+10)\left(c_{2}^{3}-c_{1}^{3}\right) \\
c_{2}^{4}= & (28-35 \alpha) c_{1}^{3}+(29 \alpha-1) c_{2}^{3}-c_{1}^{3} c_{3}^{0}-\left(c_{1}^{1} c_{3}^{1}+c_{1}^{1} c_{3}^{2}\right) \frac{\Gamma(3 q+1)}{\Gamma(q+1) \Gamma(2 q+1)}-c_{1}^{0} c_{3}^{3} \\
c_{3}^{4}= & {\left[-\frac{8+\alpha}{3}\right] c_{3}^{3}+c_{1}^{3} c_{2}^{0}+\left(c_{1}^{2} c_{2}^{1}+c_{1}^{1} c_{2}^{2}\right) \frac{\Gamma(3 q+1)}{\Gamma(q+1) \Gamma(2 q+1)}+c_{1}^{0} c_{2}^{3} }
\end{aligned}\right. \\
\left\{\begin{aligned}
c_{1}^{5}= & (25 \alpha+10)\left(c_{2}^{4}-c_{1}^{4}\right) \\
c_{2}^{5}= & (28-35 \alpha) c_{1}^{4}+(29 \alpha-1) c_{2}^{4}-c_{1}^{4} c_{3}^{0}-\left(c_{1}^{3} c_{3}^{1}+c_{1}^{1} c_{3}^{3}\right) \frac{\Gamma(4 q+1)}{\Gamma(q+1) \Gamma(3 q+1)}- \\
& c_{1}^{2} c_{3}^{2} \frac{\Gamma(4 q+1)}{\Gamma^{2}(2 q+1)}-c_{1}^{0} c_{3}^{4} \\
c_{3}^{5}= & {\left[-\frac{8+\alpha}{3}\right] c_{3}^{4}+c_{1}^{4} c_{2}^{0}+\left(c_{1}^{3} c_{2}^{1}+c_{1}^{1} c_{2}^{3}\right) \frac{\Gamma(4 q+1)}{\Gamma(q+1) \Gamma(3 q+1)}+c_{1}^{2} c_{2}^{2} \frac{\Gamma(4 q+1)}{\Gamma^{2}(2 q+1)}+c_{1}^{0} c_{2}^{4} }
\end{aligned}\right. \\
\left\{\begin{aligned}
c_{1}^{6}= & (25 \alpha+10)\left(c_{2}^{5}-c_{1}^{5}\right) \\
c_{2}^{6}= & (28-35 \alpha) c_{1}^{5}+(29 \alpha-1) c_{2}^{5}-c_{1}^{5} c_{3}^{0}-\left(c_{1}^{4} c_{3}^{1}+c_{1}^{1} c_{3}^{4}\right) \frac{\Gamma(5 q+1)}{\Gamma(q+1) \Gamma(4 q+1)}- \\
& \left(c_{1}^{3} c_{3}^{2}+c_{1}^{2} c_{3}^{3}\right) \frac{\Gamma(5 q+1)}{\Gamma(2 q+1) \Gamma(3 q+1)}-c_{1}^{0} c_{3}^{5} \\
c_{3}^{6}= & {\left[-\frac{8+\alpha}{3}\right] c_{3}^{5}+c_{1}^{5} c_{2}^{0}+\left(c_{1}^{4} c_{2}^{1}+c_{1}^{1} c_{2}^{4}\right) \frac{\Gamma(5 q+1)}{\Gamma(q+1) \Gamma(4 q+1)}+\left(c_{1}^{3} c_{2}^{2}+c_{1}^{2} c_{2}^{3}\right) } \\
& \frac{\Gamma(5 q+1)}{\Gamma(2 q+1) \Gamma(3 q+1)}+c_{1}^{0} c_{2}^{5}
\end{aligned}\right.
\end{gathered}
$$

The solution to the system equation can be expressed as:

$$
x_{j}(t)=c_{j}^{0}+c_{j}^{1} \frac{\left(t-t_{0}\right)^{q}}{\Gamma(q+1)}+c_{j}^{2} \frac{\left(t-t_{0}\right)^{2 q}}{\Gamma(2 q+1)}+c_{j}^{3} \frac{\left(t-t_{0}\right)^{3 q}}{\Gamma(3 q+1)}+c_{j}^{4} \frac{\left(t-t_{0}\right)^{4 q}}{\Gamma(4 q+1)}+c_{j}^{5} \frac{\left(t-t_{0}\right)^{5 q}}{\Gamma(5 q+1)}+c_{j}^{6} \frac{\left(t-t_{0}\right)^{6 q}}{\Gamma(6 q+1)}
$$


where $j=1,2,3$. Thus, when $q 1=q 2=q 3=0.96, \alpha=0, h=0.001$, the phase space diagram of each plane is shown in Figure 9.

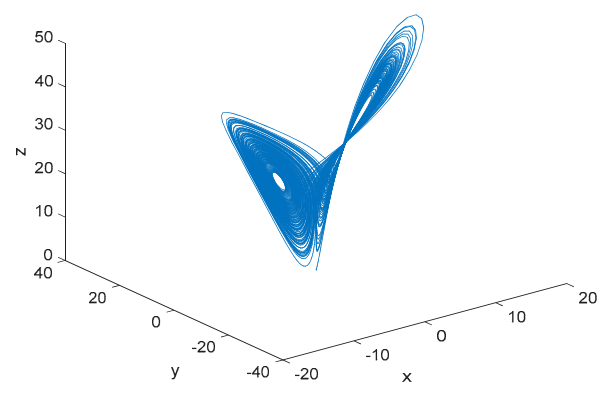

(a)

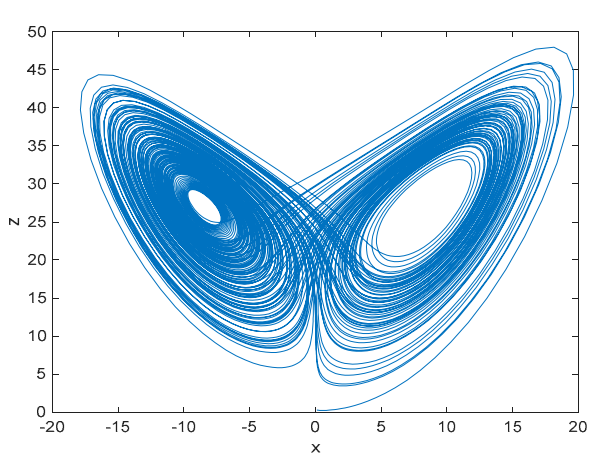

(c)

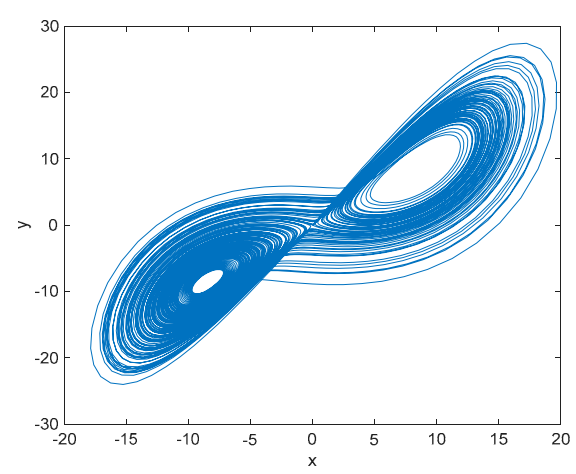

(b)

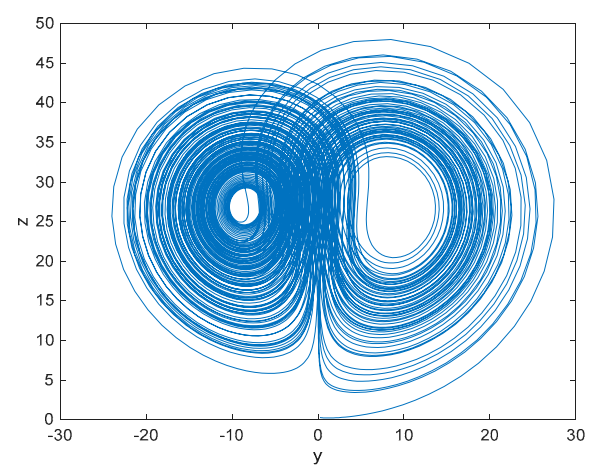

(d)

Figure 9. Phase space diagram of each plane. (a) $x-y-z$ plane; (b) $x-y$ plane; (c) $x-z$ plane; (d) $y-z$ plane.

\subsection{The Improved Adomian Decomposition Method}

The algorithm is further optimized according to the Adomian decomposition method [21].

The fractional-order unified chaotic system is taken as an example to decompose the nonlinear part. According to the given conditions, the following equation can be obtained:

$$
\left\{\begin{array}{l}
x_{1}^{0}=x_{1}\left(t_{0}\right) \\
x_{2}^{0}=x_{2}\left(t_{0}\right) \\
x_{3}^{0}=x_{3}\left(t_{0}\right)
\end{array}\right.
$$

Make $c_{1}^{0}=x_{1}^{0}, c_{2}^{0}=x_{2}^{0}, c_{3}^{0}=x_{3}^{0}$, the following equation can be obtained:

$$
\left\{\begin{array}{l}
x_{1}^{1}=\left[(25 \alpha+10)\left(c_{2}^{0}-c_{1}^{0}\right)\right] \frac{\left(t-t_{0}\right)^{q}}{q} \\
x_{2}^{1}=\left[(28-35 \alpha) c_{1}^{0}-c_{1}^{0} c_{3}^{0}+(29 \alpha-1) c_{2}^{0}\right] \frac{\left(t-t_{0}\right)^{q}}{q} \\
x_{3}^{1}=\left[c_{1}^{0} c_{2}^{0}-(8+\alpha) c_{3}^{0} / 3\right] \frac{\left(t-t_{0}\right)^{q}}{q}
\end{array}\right.
$$

From the iteration relation, the other five coefficients of $x$ can be deduced as follows:

$$
\left\{\begin{array}{l}
c_{1}^{2}=(25 \alpha+10)\left(c_{2}^{1}-c_{1}^{1}\right) \\
c_{2}^{2}=(28-35 \alpha) c_{1}^{1}+(29 \alpha-1) c_{2}^{1}-c_{1}^{1} c_{3}^{0}-c_{1}^{0} c_{3}^{1} \\
c_{3}^{2}=\left[-\frac{8+\alpha}{3}\right] c_{3}^{1}+c_{1}^{1} c_{2}^{0}+c_{1}^{0} c_{2}^{1}
\end{array}\right.
$$




$$
\begin{gathered}
\left\{\begin{array}{l}
c_{1}^{3}=(25 \alpha+10)\left(c_{2}^{2}-c_{1}^{2}\right) \\
c_{2}^{3}=(28-35 \alpha) c_{1}^{2}+(29 \alpha-1) c_{2}^{2}-c_{1}^{2} c_{3}^{0}-2 c_{1}^{1} c_{3}^{1}-c_{1}^{0} c_{3}^{2} \\
c_{3}^{3}=\left[-\frac{8+\alpha}{3}\right] c_{3}^{2}+c_{1}^{2} c_{2}^{0}+2 c_{1}^{1} c_{2}^{1}+c_{1}^{0} c_{2}^{2}
\end{array}\right. \\
\left\{\begin{array}{l}
c_{1}^{4}=(25 \alpha+10)\left(c_{2}^{3}-c_{1}^{3}\right) \\
c_{2}^{4}=(28-35 \alpha) c_{1}^{3}+(29 \alpha-1) c_{2}^{3}-c_{1}^{3} c_{3}^{0}-3\left(c_{1}^{2} c_{3}^{1}+c_{1}^{1} c_{3}^{2}\right)-c_{1}^{0} c_{3}^{3} \\
c_{3}^{4}=\left[-\frac{8+\alpha}{3}\right] c_{3}^{3}+c_{1}^{3} c_{2}^{0}+3\left(c_{1}^{2} c_{2}^{1}+c_{1}^{1} c_{2}^{2}\right)+c_{1}^{0} c_{2}^{3}
\end{array}\right. \\
\left\{\begin{array}{l}
c_{1}^{5}=(25 \alpha+10)\left(c_{2}^{4}-c_{1}^{4}\right) \\
c_{2}^{5}=(28-35 \alpha) c_{1}^{4}+(29 \alpha-1) c_{2}^{4}-c_{1}^{4} c_{3}^{0}-4\left(c_{1}^{3} c_{3}^{1}+c_{1}^{1} c_{3}^{3}\right)-6 c_{1}^{2} c_{3}^{2}-c_{1}^{0} c_{3}^{4} \\
c_{3}^{5}=\left[-\frac{8+\alpha}{3}\right] c_{3}^{4}+c_{1}^{4} c_{2}^{0}+4\left(c_{1}^{3} c_{2}^{1}+c_{1}^{1} c_{2}^{3}\right)+6 c_{1}^{2} c_{2}^{2}+c_{1}^{0} c_{2}^{4}
\end{array}\right. \\
\left\{\begin{array}{l}
c_{1}^{6}=(25 \alpha+10)\left(c_{2}^{5}-c_{1}^{5}\right) \\
c_{2}^{6}=(28-35 \alpha) c_{1}^{5}+(29 \alpha-1) c_{2}^{5}-c_{1}^{5} c_{3}^{0}-5\left(c_{1}^{4} c_{3}^{1}+c_{1}^{1} c_{3}^{4}\right) 10\left(c_{1}^{3} c_{3}^{2}+c_{1}^{2} c_{3}^{3}\right)-c_{1}^{0} c_{3}^{5} \\
c_{3}^{6}=\left[-\frac{8+\alpha}{3}\right] c_{3}^{5}+c_{1}^{5} c_{2}^{0}+5\left(c_{1}^{4} c_{2}^{1}+c_{1}^{1} c_{2}^{4}\right)+10\left(c_{1}^{3} c_{2}^{2}+c_{1}^{2} c_{2}^{3}\right)+c_{1}^{0} c_{2}^{5}
\end{array}\right.
\end{gathered}
$$

The solution to the system equation can be expressed as:

$$
x_{j}(t)=c_{j}^{0}+c_{j}^{1} \frac{\left(t-t_{0}\right)^{q}}{1 ! q}+c_{j}^{2} \frac{\left(t-t_{0}\right)^{2 q}}{2 ! q^{2}}+c_{j}^{3} \frac{\left(t-t_{0}\right)^{3 q}}{3 ! q^{3}}+c_{j}^{4} \frac{\left(t-t_{0}\right)^{4 q}}{4 ! q^{4}}+c_{j}^{5} \frac{\left(t-t_{0}\right)^{5 q}}{5 ! q^{5}}+c_{j}^{6} \frac{\left(t-t_{0}\right)^{6 q}}{6 ! q^{6}}
$$

It can be seen from the above calculation process that the improved Adomian decomposition method has no Gamma function in the process of decomposition, so the decomposition process is simpler than the Adomian decomposition method, which it is more convenient in the programming of the program and has little calculation amount.

When $q 1=q 2=q 3=0.96, \alpha=0, h=0.001$, the phase space diagram is shown in Figure 10.

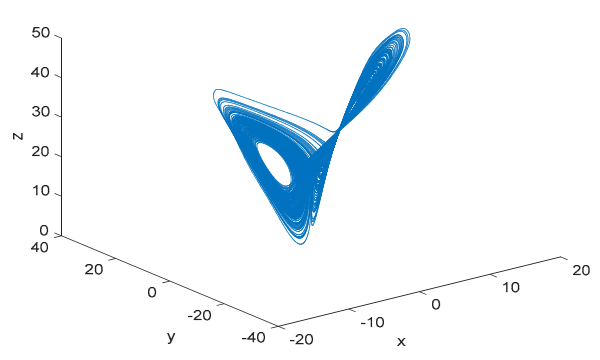

(a)

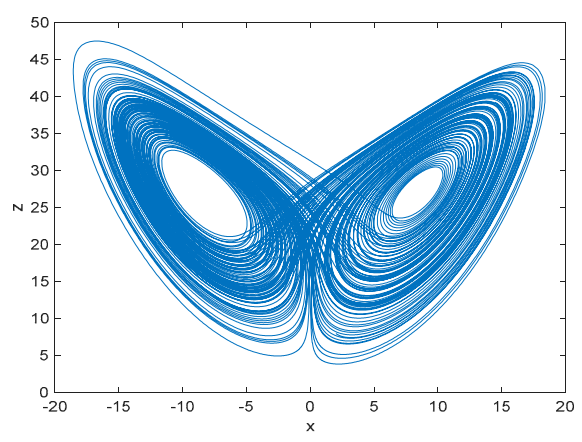

(c)

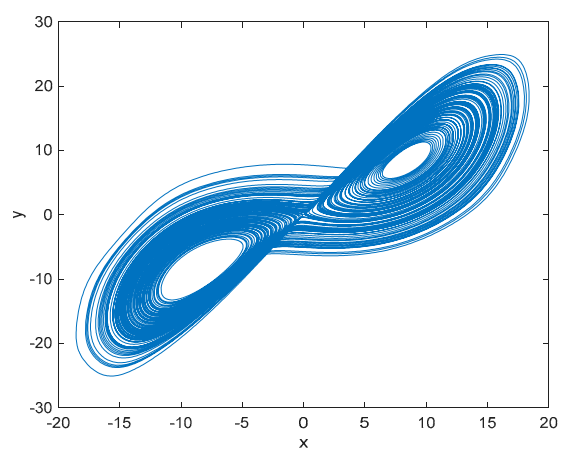

(b)

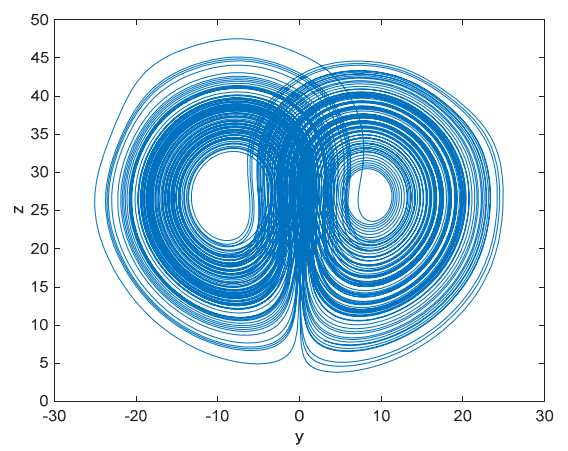

(d)

Figure 10. Phase space diagram of each plane. (a) $x-y-z$ plane; (b) $x-y$ plane; (c) $x-z$ plane; (d) $y-z$ plane. 
For the convenience of comparison, Table 1 can obtain the calling function method, the Adams prediction-correction method, the Adomian decomposition method, and the improved Adomian decomposition method performance comparison results by running the program and timing. From the results, the improved Adomian decomposition method works best.

The above three methods are directly calculated in the time domain, avoiding the approximation error caused by transforming time domain into frequency domain and improving the accuracy compared with the calling function method.

Table 1. Performance comparison of different algorithms.

\begin{tabular}{ccccc}
\hline & $\begin{array}{c}\text { Calling Function } \\
\text { Method }\end{array}$ & $\begin{array}{c}\text { Adams Prediction- } \\
\text { Correction Method }\end{array}$ & $\begin{array}{c}\text { Adomian } \\
\text { Decomposition Method }\end{array}$ & $\begin{array}{c}\text { Improved Adomian } \\
\text { Decomposition Method }\end{array}$ \\
\hline Time Complexity & $\mathrm{O}\left(\mathrm{n}^{2}\right)$, Slowly & $\mathrm{O}\left(\mathrm{n}^{2}\right)$, Slowly & $\mathrm{O}(\mathrm{n})$, Quickly & O(n), Quickly \\
Space Complexity & $\mathrm{O}(\mathrm{n})$, Complex & $\mathrm{O}(\mathrm{n})$, Complex & $\mathrm{O}(1)$, Simple & O(1), Simple \\
$\mathrm{N}=1000$ & $1.823 \mathrm{~s}$ & $1.298 \mathrm{~s}$ & $0.487 \mathrm{~s}$ & $0.306 \mathrm{~s}$ \\
$\mathrm{~N}=2000$ & $5.032 \mathrm{~s}$ & $4.680 \mathrm{~s}$ & $0.510 \mathrm{~s}$ & $0.403 \mathrm{~s}$ \\
$\mathrm{~N}=5000$ & $17.897 \mathrm{~s}$ & $15.362 \mathrm{~s}$ & $0.858 \mathrm{~s}$ & $0.623 \mathrm{~s}$ \\
$\mathrm{~N}=10,000$ & $31.259 \mathrm{~s}$ & $28.191 \mathrm{~s}$ & $1.458 \mathrm{~s}$ & $0.919 \mathrm{~s}$ \\
\hline
\end{tabular}

\section{Circuit Simulation of Fractional-Order Unified Chaotic System}

In practical engineering application, there are many algorithms for solving fractional differential equations. The most commonly used is time domain and complex frequency domain transformation method. By solving transfer function $\frac{1}{s^{q}}$, its expanded form is obtained and then transformed to time domain for solution. In reference [22], the expansion of $\frac{1}{s^{q}}$ is determined by Bode graphic approximation method, and the $\frac{1}{s^{q}}$ expansion of $q$ from 0.1 to 0.9 is derived in reference [23]. Generally, when designing a fractional-order chaotic circuit, the corresponding integer order chaotic circuit should be designed firstly, and the capacitance in the integer circuit is replaced by a fractional-order module to form a fractional-order chaotic circuit [24].

The key point of designing fractional-order chaotic circuit is the circuit design of the integral operator in the circuit diagram. Because the integral operator of $q$ can be described in the frequency domain by the transfer function $\mathrm{F}(\mathrm{s})=\frac{1}{s^{q}}$, the following unit circuit can be used to achieve the fractional order equivalent circuit [25], as shown in Figure 11.

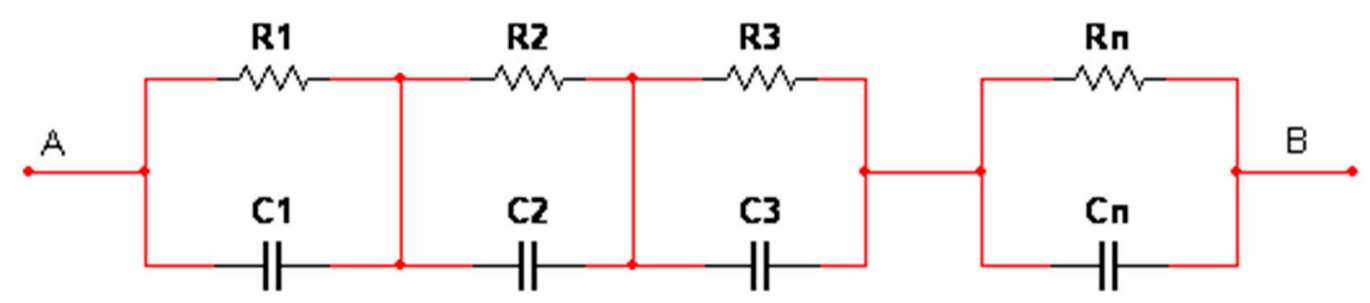

Figure 11. Equivalent circuit of fractional integral operator $\frac{1}{5^{q}}$.

The system function [26] of the equivalent circuit between A and B can be expressed as:

$$
H(s)=\frac{R_{1}}{R_{1} C_{1}}+\frac{R_{2}}{R_{2} C_{2}}+\frac{R_{3}}{R_{3} C_{3}}+\cdots+\frac{R_{n}}{R_{n} C_{n}}
$$

Use Tables 2 and 3 to calculate the cell resistance and capacitance values for the fractional $\frac{1}{s^{q}}$ property. 
Table 2. Resistance value in $q$-order fractional unit circuit.

\begin{tabular}{ccccccc}
\hline$\frac{1}{s^{q}}$ & $\mathbf{R}_{1} / \mathbf{M} \boldsymbol{\Omega}$ & $\mathbf{R}_{2} / \mathbf{M} \boldsymbol{\Omega}$ & $\mathbf{R}_{3} / \mathbf{M} \boldsymbol{\Omega}$ & $\mathbf{R}_{4} / \mathbf{M} \boldsymbol{\Omega}$ & $\mathbf{R}_{5} / \mathbf{M} \boldsymbol{\Omega}$ & $\mathbf{R}_{6} / \mathbf{M} \boldsymbol{\Omega}$ \\
\hline$\frac{1}{s^{0.1}}$ & 0.636 & 0.3815 & 0.5672 & & & \\
$\frac{1}{s^{0.2}}$ & 1.130 & 0.6070 & 0.3500 & 0.425 & 0.2498 & \\
$\frac{1}{s^{0.3}}$ & 2.050 & 0.9380 & 0.4830 & 0.262 & 0.1430 & 0.106 \\
$\frac{1}{s^{0.4}}$ & 3.744 & 1.3920 & 0.6310 & 0.294 & 0.123. & 0.068 \\
$\frac{1}{s^{0.5}}$ & 6.824 & 1.9440 & 0.7440 & 0.296 & 0.0754 & 0.030 \\
$\frac{1}{s^{0.6}}$ & 12.330 & 2.44480 & 0.7380 & 0.233 & 0.0754 & 0.006 \\
$\frac{1}{s^{0.7}}$ & 21.900 & 2.6000 & 0.5260 & 0.113 & 0.0246 & \\
$\frac{1}{s^{0.8}}$ & 37.850 & 1.7540 & 0.1700 & 0.017 & 0.0018 & \\
$\frac{1}{s^{0.9}}$ & 62.840 & 0.2500 & 0.0025 & & & \\
\hline
\end{tabular}

Table 3. Capacitance value in $q$-order fractional unit circuit.

\begin{tabular}{ccccccc}
\hline$\frac{1}{s^{q}}$ & $\mathrm{C}_{1} / \mu \mathrm{F}$ & $\mathrm{C}_{2} / \mu \mathrm{F}$ & $\mathrm{C}_{3} / \mu \mathrm{F}$ & $\mathrm{C}_{4} / \mu \mathrm{F}$ & $\mathrm{C}_{5} / \mu \mathrm{F}$ & $\mathrm{C}_{6} / \mu \mathrm{F}$ \\
\hline$\frac{1}{s^{0.1}}$ & 15.720 & 0.1572 & 0.0006335 & & & \\
$\frac{1}{s^{0.2}}$ & 27.990 & 2.9300 & 0.285 & 0.0132 & & \\
$\frac{1}{s^{0.3}}$ & 22.640 & 5.5200 & 1.200 & 0.2460 & 0.029 & \\
$\frac{1}{s^{0.4}}$ & 15.020 & 5.9260 & 1.920 & 0.6050 & 0.183 & 0.036 \\
$\frac{1}{s^{0.5}}$ & 9.246 & 5.1450 & 2.129 & 0.8480 & 0.324 & 0.925 \\
$\frac{1}{s^{0.6}}$ & 5.527 & 4.0850 & 1.990 & 0.9260 & 0.420 & 0.156 \\
$\frac{1}{s_{0.7}^{0.7}}$ & 3.284 & 3.1390 & 1.700 & 0.8860 & 0.454 & 0.207 \\
$\frac{1}{s^{0.8}}$ & 1.980 & 2.4000 & 1.390 & 0.7800 & 0.420 & \\
$\frac{1}{s^{0.9}}$ & 1.232 & 1.8400 & 1.100 & & & \\
\hline
\end{tabular}

The circuit diagram of the fractional-order unified chaotic system is shown in Figure 12.



Figure 12. Fractional-order unified chaotic system circuit diagram.

For fractional-order unified chaotic system, the linear part is composed of basic operational amplifier circuit, such as reverse proportional amplifier, reverse adder, subtracter, in-phase proportional 
amplifier, reverse integrator, etc., and the nonlinear term is represented by multiplier. The parameters of each component are as follows:

$$
\left\{\begin{array}{l}
R 1=R 8=R 20=R 21=10 K \Omega \\
R 2=R 9=35 K \Omega \\
R 4=R 15=R 19=R 22=R 23=R 27=100 K \Omega \\
R 10=10.15 K \Omega \\
R 11=29 K \Omega \\
R 14=10.35 K \Omega \\
R 29=30 K \Omega
\end{array}\right.
$$

And when $\alpha=0.5$,

$$
\left\{\begin{array}{l}
R 3=22.5 K \Omega \\
R 12=20 K \Omega \\
R 13=58 K \Omega \\
R 28=8.5 K \Omega
\end{array}\right.
$$

When $q 1=q 2=q 3=0.9$, use Tables 2 and 3 to calculate:

$$
\left\{\begin{array}{l}
R 5=R 16=R 24=1.55 M \Omega \\
R 6=R 17=R 25=6154 M \Omega \\
R 7=R 18=R 26=2.5 M \Omega \\
C 1=C 2=C 7=0.73 \mu F \\
C 3=C 5=C 8=0.52 \mu F \\
C 4=C 6=C 9=1.1 \mu F
\end{array}\right.
$$

The simulation circuit diagram of the fractional-order unified chaotic system is built in the Multisim 14.1 version. The simulation result is shown in Figure 13.

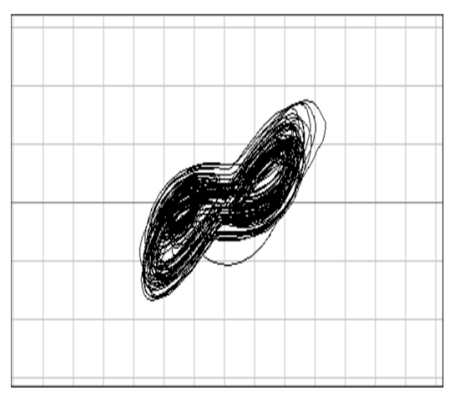

(a)

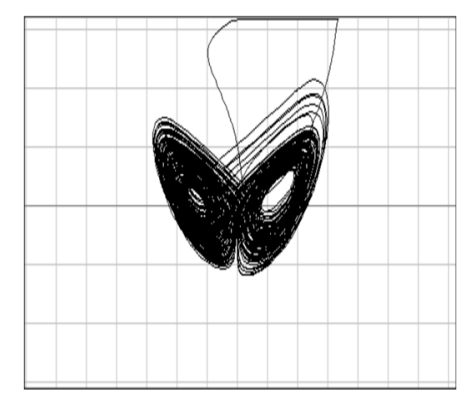

(b)

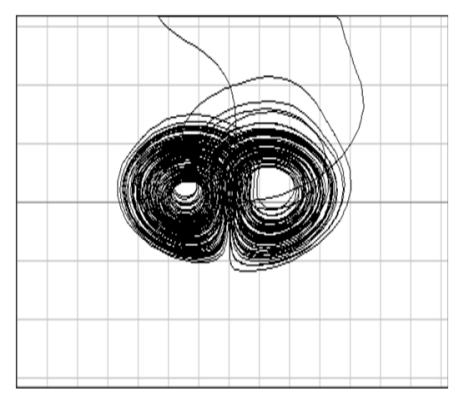

(c)

Figure 13. Circuit simulation diagram. (a) $x$-y plane; (b) $x$-z plane; (c) $y$-z plane.

It can be seen from Figure 13 that the circuit simulation diagram of the fractional-order unified chaotic system is basically consistent with the phase space diagram obtained by the numerical solution of the system, and it is verified that the 0.9-order fractional-order unified chaotic system is exists.

\section{Control and Synchronization of Fractional-Order Unified Chaotic System}

\subsection{Control of Fractional-Order Unified Chaotic System}

The controlled model of the fractional-order unified chaotic system is:

$$
\left\{\begin{array}{l}
D^{q 1} x_{1}=(25 \alpha+10)\left(x_{2}-x_{1}\right)+u_{1} \\
D^{q 2} x_{2}=(28-35 \alpha) x_{1}-x_{1} x_{3}+(29 \alpha-1) x_{2}+u_{2} \\
D^{q 3} x_{3}=x_{1} x_{2}-\frac{(8+\alpha) x_{3}}{3}+u_{3}
\end{array}\right.
$$


Select:

$$
\left\{\begin{array}{l}
u_{1}=v_{1} \\
u_{2}=x_{1} x_{3}+v_{2} \\
u_{3}=-x_{1} x_{2}+v_{3}
\end{array}\right.
$$

where, $u_{1}, u_{2}, u_{3}$ are the control input, $v_{1}, v_{2}, v_{3}$ are the linear functions of $x_{1}, x_{2}, x_{3}$.

Get the following formula:

$$
\left\{\begin{array}{l}
D^{q 1} x_{1}=(25 \alpha+10)\left(x_{2}-x_{1}\right)+v_{1} \\
D^{q 2} x_{2}=(28-35 \alpha) x_{1}+(29 \alpha-1) x_{2}+v_{2} \\
D^{q 3} x_{3}=-\frac{(8+\alpha) x_{3}}{3}+v_{3}
\end{array}\right.
$$

To ensure stability, select $v_{1}, v_{2}, v_{3}$, respectively:

$$
\left\{\begin{array}{l}
v_{1}=-k_{1} x_{1} \\
v_{2}=k_{2} x_{2} \\
v_{3}=0
\end{array}\right.
$$

where $k_{1}, k_{2}$ is the gain, and the system becomes:

$$
\left\{\begin{array}{l}
D^{q 1} x_{1}=(25 \alpha+10)\left(x_{2}-x_{1}\right)-k_{1} x_{1} \\
D^{q 2} x_{2}=(28-35 \alpha) x_{1}+(29 \alpha-1) x_{2}-k_{2} x_{2} \\
D^{q 3} x_{3}=-\frac{(8+\alpha) x_{3}}{3}
\end{array}\right.
$$

The system is written in matrix form as:

$$
\begin{gathered}
D^{q} x=A x \\
A=\left[\begin{array}{ccc}
-25 \alpha-10-k_{1} & 25 \alpha+10 & 0 \\
28-35 \alpha & 29 \alpha-1-k_{2} & 0 \\
0 & 0 & -(\alpha+8) / 3
\end{array}\right]
\end{gathered}
$$

The characteristic polynomial is:

$$
\begin{aligned}
& \operatorname{Det}(\lambda E-A)=\left[\left(\lambda+25 \alpha+10+k_{1}\right)\left(\lambda-29 \alpha+1+k_{2}\right)-(25 \alpha+10)(28-35 \alpha)\right] \\
& =\lambda^{2}-29 \lambda \alpha+\lambda+\lambda k_{2}+25 \lambda-725 \alpha^{2}+25 \alpha+25 \alpha k_{2}+10 \lambda-290 \alpha+10+10 k_{2}+\lambda k_{1}- \\
& 29 \alpha k_{1}+k_{1}+k_{1} k_{2}-700 \alpha+875 \alpha^{2}-280+350 \alpha
\end{aligned}
$$

Collation can be obtained as follows:

$$
\begin{aligned}
& \operatorname{Det}(\lambda E-A)=(\lambda+(\alpha+8) / 3) \times\left[\lambda^{2}+\left(k_{1}+k_{2}-4 \alpha+11\right) \lambda+150 \alpha^{2}+\left(-29 k_{1}+25 k_{2}-615\right) \alpha+\right. \\
& \left.\left(k_{1}+10\right)\left(k_{2}+1\right)-280\right]
\end{aligned}
$$

According to the lemma [27] $\frac{d^{q} x}{d t q}=A x, 0<q<1$, if any eigenvalues of the matrix $A$ meet $|\arg (\operatorname{eig}(A))|>\frac{q \pi}{2}$, and the system is asymptotically stable. When the eigenvalues of the matrix are negative, the above research system is stable. So, the solution to $k_{1}, k_{2}$ is worth noting that the eigenvalue is negative. The correlation properties of the matrix eigenvalues can be used to the following inequalities:

$$
\begin{aligned}
& \left\{\begin{array}{l}
k_{1}+k_{2} \geq 4 \alpha-11 \\
29 k_{1}-25 k_{2} \leq-615 \\
\left(k_{1}+10\right)\left(k_{2}+1\right) \geq 280
\end{array}\right. \\
& \left\{\begin{array}{l}
k_{1}+k_{2} \geq 4 \alpha-11 \\
29 k_{1}-25 k_{2} \geq-315 \\
k_{1} k_{2}-28 k_{1}+35 k_{2} \geq 735
\end{array}\right.
\end{aligned}
$$


To solve the inequality system, we can get:

$$
\left\{\begin{array}{l}
k_{1}=0 \\
k_{2}=27
\end{array}\right.
$$

and

$$
\left\{\begin{array}{l}
k_{1}=10 \\
k_{2}=23
\end{array}\right.
$$

When $q 1=q 2=q 3=0.9$ and $h=0.001$, the control effect is shown in Figure 14. Figure 14 shows that the three control laws can make the state of fractional-order unified chaotic system asymptotically approach zero.

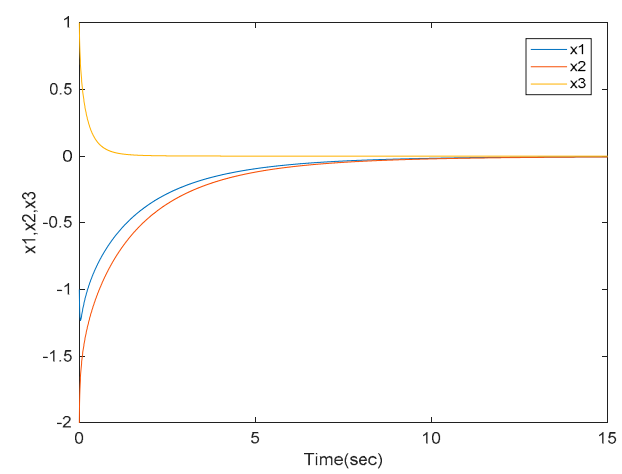

(a)

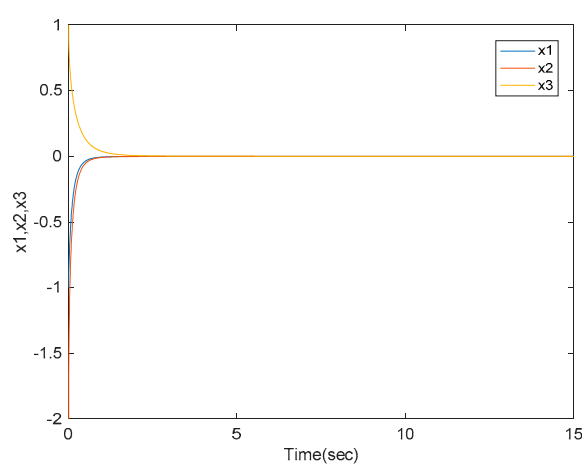

(b)

Figure 14. Control effect diagram. (a) $\alpha=1, k_{1}=10, k_{2}=23$; (b) $\alpha=0, k_{1}=10, k_{2}=23$.

\subsection{Synchronization of Fractional-Order Unified Chaotic System}

The response system is:

$$
\left\{\begin{array}{l}
D^{q 1} y_{1}=(25 \beta+10)\left(y_{2}-y_{1}\right)+u_{1} \\
D^{q 2} y_{2}=(28-35 \beta) y_{1}-y_{1} y_{3}+(29 \beta-1) y_{2}+u_{2} \\
D^{q 3} y_{3}=y_{1} y_{2}-\frac{(8+\beta) y_{3}}{3}+u_{3}
\end{array}\right.
$$

The error between the response system and the drive system is:

$$
\left\{\begin{array}{l}
e_{1}=y_{1}-x_{1} \\
e_{2}=y_{2}-x_{2} \\
e_{3}=y_{3}-x_{3}
\end{array}\right.
$$

The dynamic equation of the error system is obtained as follows:

$$
\left\{\begin{aligned}
D^{q 1} e_{1}= & (25 \beta+10)\left(e_{2}-e_{1}\right)+25(\beta-\alpha)\left(x_{2}-x_{1}\right)+u_{1} \\
D^{q 2} e_{2}= & (28-35 \beta) e_{1}+(29 \beta-1) e_{2}-35(\beta-\alpha) x_{1}+e_{1} e_{3}-e_{1} y_{3}- \\
& e_{3} y_{1}+29(\beta-\alpha) x_{2}+u_{2} \\
D^{93} e_{3}= & -\frac{(8+\beta)}{3} e_{3}-\frac{(\beta-\alpha)}{3} x_{3}-e_{1} e_{2}+e_{1} y_{2}+e_{2} y_{1}+u_{3}
\end{aligned}\right.
$$

Since it is the same fractional chaotic system, the selected gain $k_{1}$ and $k_{2}$ can be used. When $\alpha=1$, $\beta=0.5, k_{1}=10, k_{2}=23$, the solid line represents the variation curve of the drive system state variable $x(t)$, and the broken line represents the variation curve of the response system $y(t)$. The synchronization effect diagram is shown in Figure 15. The control input diagram and the synchronization error diagram are shown in Figure 16. 

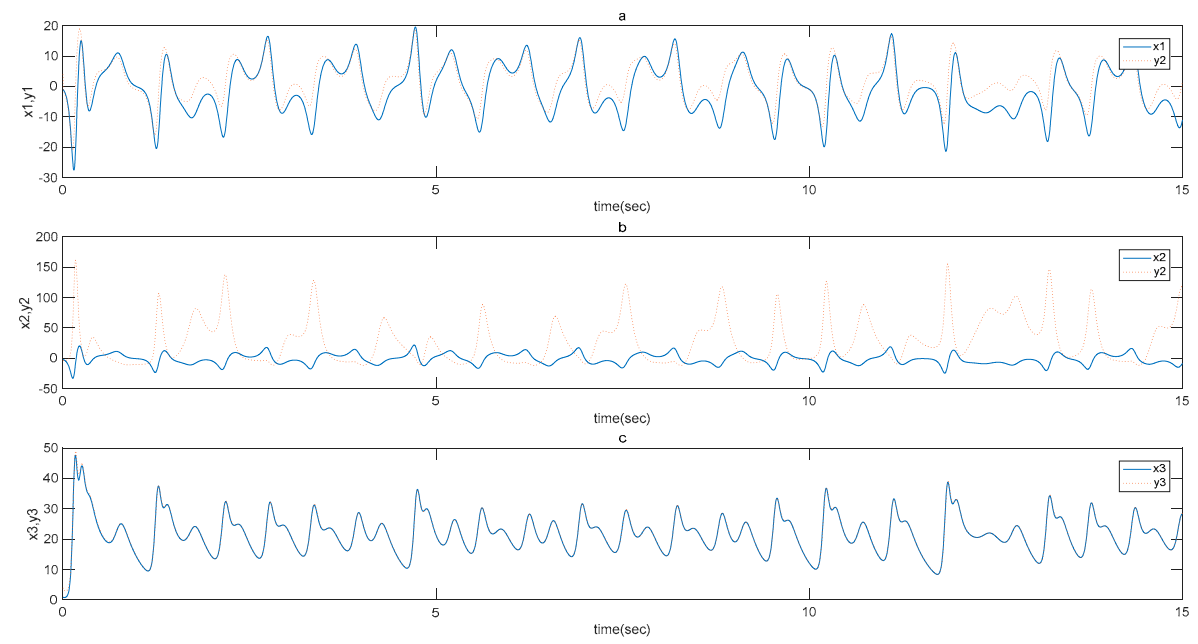

Figure 15. Synchronization effect diagram.

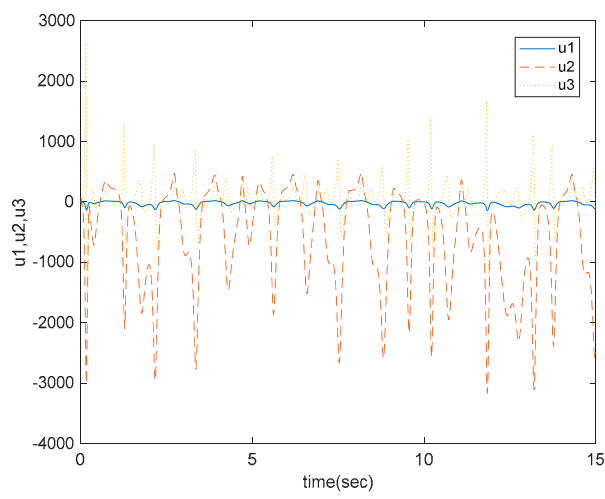

(a)

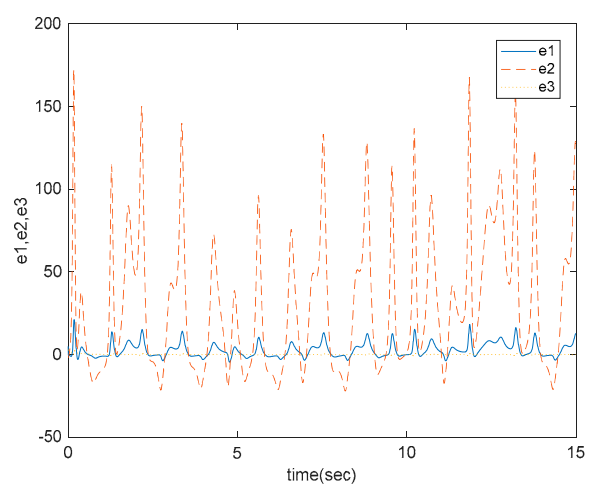

(b)

Figure 16. Control input and error diagram. (a) Control input diagram; (b) Error diagram.

Figures 15 and 16 show that under the same control law, the response system of the fractional-order unified chaotic system is synchronized with the drive system, and has good control performance.

\section{Conclusions}

In this paper, different numerical methods of fractional calculus are used to analyze the fractional-order unified chaotic system, that is the Adams prediction-correction method, the Adomian decomposition method and the improved Adomian decomposition method. Secondly, 0.9-order fractional-order unified chaotic circuit simulation diagram is designed. Finally, the control and synchronization of the fractional-order unified chaotic system is completed by the active control method.

Author Contributions: G.L., X.Z., and H.Y. conceived the idea and research theme. X.Z. designed and performed the experiments. G.L., X.Z., and H.Y. analyzed the experimental results. G.L., X.Z., and H.Y. wrote and revised the paper.

Funding: This work was supported by the National Natural Science Foundation of China (no. 51709228).

Conflicts of Interest: The authors declare no conflict of interest.

\section{References}

1. Chen, G.R.; Ueta, T. Yet another chaotic attractor. Int. J. Bifurc. Chaos 1999, 9, 1465-1466. [CrossRef]

2. Lü, J.H.; Chen, G.R.; Zhang, S.C. Dynamical analysis of a new chaotic attractor. Int. J. Bifurc. Chaos 2002, 12, 1001-1015. [CrossRef]

3. Rössler, O.E. An equation for continuous chaos. Phys. Lett. A 1976, 57, 397-398. [CrossRef] 
4. Lü, J.H.; Chen, G.R.; Cheng, D.Z. A new chaotic system and beyond: the generalized Lorenz-like system. Int. J. Bifurc. Chaos 2004, 14, 1507-1537. [CrossRef]

5. Liu, W.B.; Chen, G.R. A new chaotic system and its generation. Int. J. Bifurc. Chaos 2003, 13, $261-267$. [CrossRef]

6. Liu, C.X.; Liu, T.; Liu, L.; Liu, K. A new chaotic attractor. Chaos Solitons Fractals 2004, 22, 1031-1038. [CrossRef]

7. Qi, G.Y.; Chen, G.R.; Du, S.Z.; Chen, Z.Q.; Yuan, Z.Z. Analysis of a new chaotic system. Phys. A 2005, 352, 295-308. [CrossRef]

8. Bao, B.C.; Liu, Z.; Xu, J.P. New chaotic system and its hyperchaos generation. J. Syst. Eng. Electron. 2009, 20, 1179-1187.

9. Chen, X.R.; Liu, C.X.; Wang, F.Q.; Li, Y.X. Study on the fractional-order Liu chaotic system with circuit experiment and its control. Acta Phys. Sin. 2008, 57, 1416-1422.

10. Wang, F.Q.; Liu, C.X. Study on the critical chaotic system with fractional order and circuit experiment. Acta Phys. Sin. 2006, 55, 3922-3927.

11. Mao, X.X.; Sun, K.H.; Liu, W.H. Image encryption algorithm based on fractional order unified chaotic system. Transducer Microsyst. Technol. 2017, 36, 138-141.

12. He, S.B.; Sun, K.H.; Wang, H.H. Solution of the fractional-order chaotic system based on Adomian decomposition algorithm and its complexity analysis. Acta Phys. Sin. 2014, 63, 030502.

13. Wu, C.M. Linear feedback synchronization control method for fractional order chaotic systems. Control Eng. China 2019, 26, 898-902.

14. Yan, X.M.; Shang, T.; Zhao, X.G. Synchronization of uncertain fractional-order chaotic systems based on the fractional-order sliding mode controller. Acta Math. Appl. Sin. 2018, 41, 765-776.

15. Rabah, K.; Ladaci, S.; Lashab, M. Bifurcation-based fractional-order $\mathrm{PI}^{\lambda} \mathrm{D}^{\mu}$ controller design approach for nonlinear chaotic systems. Front. Inf. Technol. Electron. Eng. 2018, 19, 180-191. [CrossRef]

16. Rajagopal, K.; Karthikeyan, A.; Srinivasan, A. Bifurcation and chaos in time delayed fractional order chaotic memfractor oscillator and its sliding mode synchronization with uncertainties. Chaos Solitons Fractals 2017, 103, 347-356. [CrossRef]

17. Tao, C.H.; Lu, J.A.; Lü, J.H. The feedback synchronization of a unified chaotic system. Acta Phys. Sin. 2002, $51,1497-1501$.

18. Wu, Q.; Huang, J.H. Fractional Calculus; Tsinghua University Press: Beijing, China, 2016.

19. Psihoyios, G.; Simos, T.E. Trigonometrically fitted Adams-Bashforth-Moulton methods for periodic initial value problems. Proceedings Second MIT Conference on Compurational Fluid and Solid Mechanics, Cambridge, MA, USA, 17-20 June 2003.

20. Zhu, Y.G.; Chang, Q.S.; Wu, S.C. A new algorithm for calculating Adomian polynomials. Appl. Math. Comput. 2005, 169, 402-416. [CrossRef]

21. Lei, T.F.; Zhang, X.; Zang, H.Y.; Xia, X.X. Experimental simulation of fractional Rabinovich hyperchaotic system based on improved Adomian decomposition method. Exp. Technol. Manag. 2018, 35, 137-140.

22. Charef, A.; Sun, H.H.; Tsao, Y.Y.; Onaral, B. Fractal system as represented by singularity function. IEEE Trans. Autom. Control 1992, 37, 1465-1470. [CrossRef]

23. Ahmad, W.M.; Sprott, J.C. Chaos in fractional-order autonomous nonlinear systems. Chaos Solitons Fractals 2003, 16, 339-351. [CrossRef]

24. Zhang, X.H.; Sun, Q. Construction of heterogeneous fractional-order chaotic system and its multiple circuits simulation. J. Syst. Simul. 2014, 26, 1460-1466.

25. Lei, T.F.; Fu, H.Y.; Zhang, X.; Wang, Z.L. Dynamics analysis and circuit implementation of a fractional-order chaotic systems based on adomian decomposition. Chin. J. Electron Devices 2019, 42, 193-200.

26. Sun, K.H.; Yang, J.L.; Qiu, S.S. Circuit simulation and implement for fractional-order chaotic systems. Comput. Simul. 2011, 28, 117-119.

27. Geng, Y.F.; Wang, L.Z. Function projective synchronization of fractional-order united chaotic system based on sliding mode control. J. Tianjin Norm. Univ. Nat. Sci. Ed. 2019, 39, 23-26.

(C) 2019 by the authors. Licensee MDPI, Basel, Switzerland. This article is an open access article distributed under the terms and conditions of the Creative Commons Attribution (CC BY) license (http://creativecommons.org/licenses/by/4.0/). 